\title{
Métodos diaǵnósticos para retinopatia induzida pelo difosfato de cloroquina nos portadores de lúpus eritematoso sistêmico
}

\author{
Diagnostic methods for chloroquine diphosphate induced retinopathy in \\ systemic lupus erythematosus
}

\author{
Luciana Duarte Rodrigues ${ }^{1}$ \\ Samuel Katsuyuki Shinjo ${ }^{2}$ \\ Maria Kiyoko Oyamada ${ }^{3}$ \\ Pedro Durães Serracarbassa ${ }^{4}$ \\ Walter Yukihiko Takahashi ${ }^{5}$ \\ Eduardo Ferreira Borba ${ }^{6}$ \\ Eloísa Silva Dutra de Oliveira Bonfá ${ }^{7}$ \\ Yoshitaka Nakashima ${ }^{8}$
}

Trabalho realizado no Hospital das Clínicas da Faculdade de Medicina da Universidade de São Paulo - USP São Paulo (SP) - Brasil.

Pós-graduanda em Medicina pela Faculdade de Medicina da Universidade de São Paulo - USP - São Paulo (SP) - Brasil.

Doutor, Médico Assistente do Serviço de Reumatologia do Hospital das Clínicas da Faculdade de Medicina da USP - São Paulo (SP) - Brasil.

Doutora, Médica Assistente do Setor de Neuroftalmologia e Órbita do Hospital das Clínicas da Faculdade de Medicina da USP - São Paulo (SP) - Brasil

${ }^{4}$ Doutor, Médico Colaborador do Setor de Retina do Hospital das Clínicas da Faculdade de Medicina da USP - São Paulo (SP) - Brasil.

${ }^{5}$ Doutor, Responsável pelo Setor de Retina do Hospital das Clínicas da Faculdade de Medicina da USP - São Paulo (SP) - Brasil.

${ }^{6}$ Livre-docente, Professor Associado da Faculdade de Medicina da USP - São Paulo (SP) - Brasil.

Livre-docente, Professora Titular e Chefe do Serviço de Reumatologia do Hospital das Clínicas da Faculdade de Medicina da USP - São Paulo (SP) - Brasil.

${ }^{8}$ Doutor, Médico Assistente do Setor de Retina do Hospital das Clínicas da Faculdade de Medicina da USP São Paulo (SP) - Brasil.

Endereço para correspondência: Luciana Duarte Rodrigues. Rua Pitangueiras, 237 - São Paulo (SP) CEP 04052-020

E-mail: rodrigues_luciana@ hotmail.com

Recebido para publicação em 10.06.2008

Última versão recebida em 10.12.2008

Aprovação em 02.04.2009

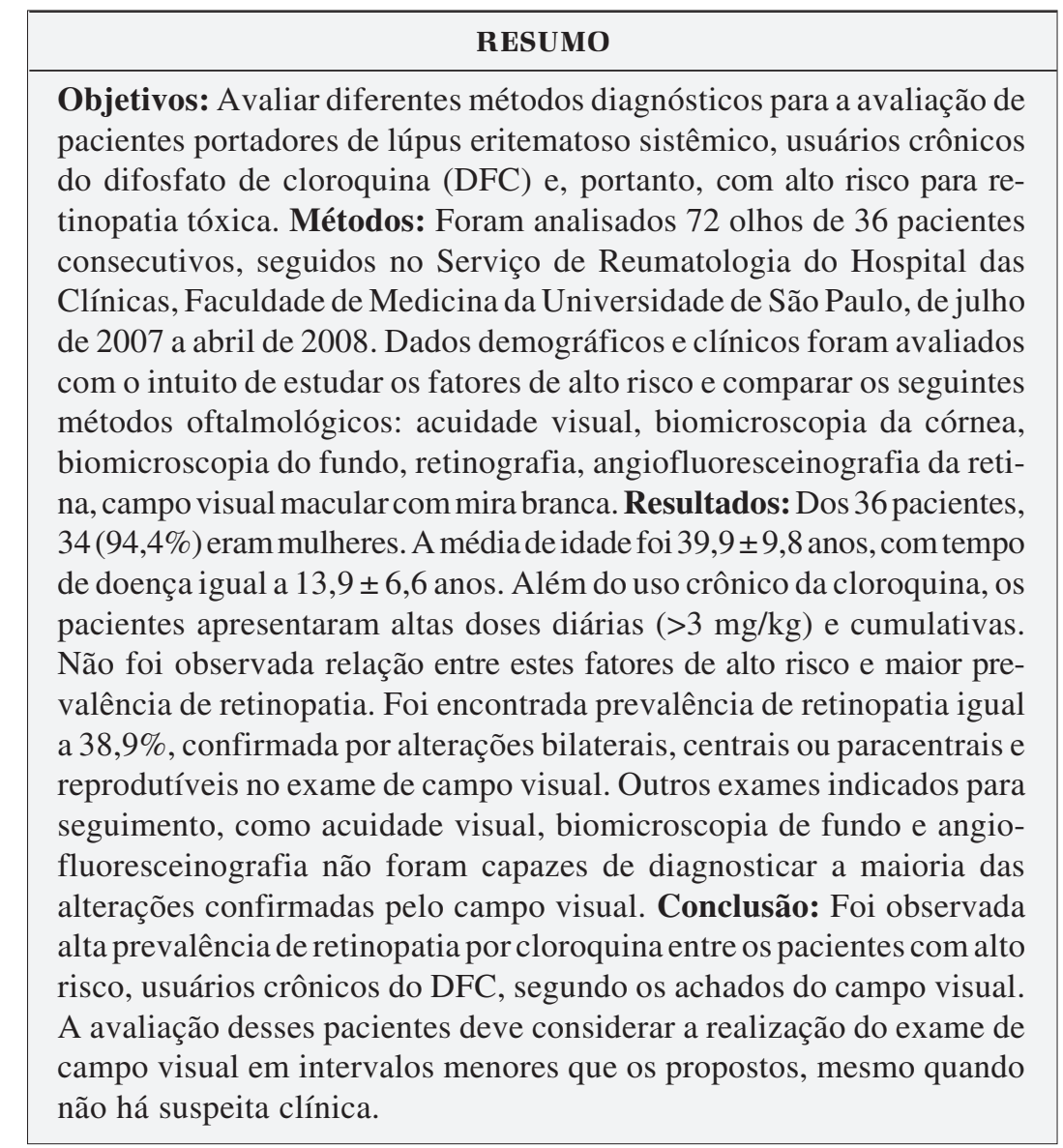

Descritores: Retina/patologia; Retina/efeito de drogas; Doenças da retina; Cloroquina/ uso terapêutico; Lúpus eritematoso sistêmico; Fatores de risco

\section{INTRODUÇÃO}

Os antimaláricos são amplamente utilizados atualmente, especialmente no tratamento do lúpus eritematoso sistêmico (LES), por apresentarem vários efeitos benéficos nestes pacientes. Auxiliam no controle da atividade da doença, minimizam a sua reativação, melhoram o perfil lipídico, diminuem eventos trombóticos e cardiovasculares e, consequentemente, diminuem a taxa de mortalidade ${ }^{(1-6)}$. Assim, uma vez estabelecido o diag- 
nóstico de LES, há uma tendência ao uso dos antimaláricos de forma contínua e prolongada.

O uso crônico dos antimaláricos pode ocasionar efeitos tóxicos oculares ${ }^{(7-9)}$. A retinopatia relacionada ao difosfato de cloroquina (DFC) é o efeito colateral mais temido por ser potencialmente irreversível ${ }^{(5-7)}$. A incidência da retinopatia tardia é $0,5 \%$, mas a retinopatia em fase precoce pode acometer $16 \%$ dos usuários ${ }^{(7)}$. É possível observar a maculopatia evoluir em estágios progressivos de acometimento da acuidade visual, alterações estruturais e funcionais. As lesões iniciais descritas são perda do reflexo foveolar, halo parafoveal discreto e palidez do epitélio pigmentado da retina $(\mathrm{EPR})^{(8)}$. A maculopatia típica em "olho de boi" é caracterizada por hiperpigmentação foveal central circundada por despigmentação e associada, nos estágios mais avançados, a redução da acuidade visual e ao campo visual tubular ${ }^{(8)}$.

Os fatores de alto risco que podem contribuir para o desenvolvimento de retinopatia induzida por antimaláricos, em particular, o DFC são: a) doses diárias acima de $3 \mathrm{mg} / \mathrm{kg} / \mathrm{dia}$, b) pacientes com alta taxa de gordura corporal, c) presença de doenças renais ou hepáticas, d) idade acima que 60 anos, e) tempo de uso acima de $5 \operatorname{anos}^{(9)}$.

Entre os fatores de risco, o último tende a ser um denominador comum em pacientes com LES crônico. Portanto, torna-se importante a avaliação oftalmológica regular dos pacientes usuários crônicos, no intuito de diagnosticar precocemente os possíveis efeitos tóxicos dos antimaláricos. Os exames recomendados, na fase inicial e para o seguimento do paciente tratado com DFC, são o mapeamento da retina e a avaliação da função macular pela tela de Amsler ${ }^{(10)}$ ou campimetria estática automatizada dos 10 graus centrais com mira branca ${ }^{(9)}$. O teste de visão de cores é opcional e indicado principalmente para a detecção prévia de discromatopsias em geral $^{(9)}$. A retinografia também é opcional e deve ser usada para documentar lesões preexistentes que possam ser confundidas com retinopatia tóxica inicial ${ }^{(11)}$. A angiofluoresceinografia é indicada apenas nos pacientes de alto risco ou com suspeita de retinopatia ${ }^{(9)}$. O eletrorretinograma multifocal deve ser realizado na fase inicial do tratamento, para a obtenção da resposta basal a ser utilizada como parâmetro nos exames subsequentes. A utilização do eletrooculograma e do eletrorretinograma de campo total no monitoramento da toxicidade macular é bastante controversa na literatura.

Torna-se interessante comparar os diferentes métodos oftalmológicos mais utilizados na prática clínica com relação à sua utilidade na população de pacientes com alto risco para retinopatia tóxica.

O objetivo do presente estudo é avaliar pacientes portadores de LES, usuários crônicos de antimaláricos, quanto a:

1) Prevalência de retinopatia por cloroquina, definida pelo achado de defeitos em dois exames de campo visual (10 graus centrais com mira branca) subsequentes;

2) Relação entre fatores de risco como dose diária, dose total cumulativa, tempo de doença e prevalência de retinopatia por cloroquina;
3) Relação entre os fatores de risco estudados e os achados nos exames: biomicroscopia da córnea, biomicroscopia do fundo, angiofluoresceinografia da retina e campo visual;

4) Relação entre acuidade visual, campo visual e os demais exames realizados.

\section{MÉTODOS}

\section{Pacientes}

Foram avaliados 80 olhos consecutivos de 40 pacientes com LES, em uso de DFC por mais de cinco anos, provenientes do Serviço de Reumatologia do Hospital das Clínicas da Faculdade de Medicina da Universidade de São Paulo (HCFMUSP), São Paulo, no período entre julho de 2007 e abril de 2008. Todos os pacientes preenchiam pelo menos quatro dos onze critérios classificatórios do LES ${ }^{(12)}$.

Os dados clínico-demográficos, laboratoriais e referentes ao uso do DFC (tempo de uso, dose diária ingerida e total cumulativa) foram obtidos através dos prontuários dos pacientes. Para o cálculo da dose ideal de DFC ingerido, foram levados em consideração o peso ideal ${ }^{(13)}$ (considerando o índice de massa corporal ideal igual a 22,0) e o peso magro (calculado pela fórmula de James) $^{(14)}$.

Foram excluídos os pacientes que apresentaram maculopatia não relacionada ao uso do DFC, opacidade importante dos meios ou ametropias acima de 5 dioptrias.

Todos os pacientes assinaram o Termo de Consentimento Livre e Esclarecido aprovado previamente pela CAPPESq.

\section{Exames oftalmológicos}

Os pacientes foram submetidos a exame oftalmológico, que consistiu na medida da acuidade visual corrigida em cada olho, em logMAR (Tabela do ETDRS), biomicroscopia da córnea em lâmpada de fenda após midríase com uma gota de colírio de tropicamida a 1\%, tonometria de aplanação de Goldmann, biomicroscopia do fundo, retinografia, angiofluoresceinografia da retina, campimetria visual 10.2 (SITA FAST, em aparelho Humphrey com mira branca, utilizando-se correção para perto). Todos estes exames foram realizados no Serviço de Oftalmologia do HC-FMUSP.

Foi considerada como alteração do campo visual a presença de dois ou mais escotomas relativos ou absolutos, adjacentes, centrais ou paracentrais, e bilaterais. Neste caso, os pacientes foram reavaliados para a confirmação das alterações, após aproximadamente dois meses de intervalo entre os exames.

\section{Análise estatística}

Para comparar acuidade visual em $\log \mathrm{MAR}$, tempo de doença e fatores de risco (tempo de uso do DFC, dose cumulativa, dose por quilograma de peso ideal, dose por quilograma de peso magro, alterações renais e hepáticas) com os resultados dos exames (normal versus alterado), foi utilizado o teste de Mann-Whitney. Para comparar os resultados do exame de 
campo visual com os achados na biomicroscopia do fundo, angiofluoresceinografia da retina e biomicroscopia da córnea, foi utilizado o teste Qui-quadrado e, quando necessário, o teste Exato de Fisher. Os valores foram considerados estatisticamente significativos quando $\mathrm{p}<0,05$.

\section{RESULTADOS}

Dos quarenta pacientes, quatro foram excluídos: um apresentou drusas maculares, outro apresentou catarata densa, e dois tinham ametropias acima de 5 dioptrias. Portanto, 36 pacientes (72 olhos) foram submetidos à avaliação completa.

A média de idade \pm desvio padrão (DP) dos pacientes foi $39,9 \pm 9,8$ anos (22 e 65 anos), com tempo de doença de 13,9 $\pm 6,6$ anos. Dos 36 pacientes, $34(94,4 \%)$ eram do sexo feminino (Tabela 1).

Quanto às manifestações clínicas, 32 (88,9\%) apresentavam acometimento mucocutâneo, 34 (94,4\%) articular, 18 $(50,0 \%)$ renal, $2(5,6 \%)$ neurológico, $8(22,2 \%)$ serosite e 13 $(36,1 \%)$ hematológico. Dos 36 pacientes, um apresentava insuficiência renal crônica dialítica, e não houve caso de pacientes com hepatopatia.

Metade dos pacientes fazia uso de corticoterapia (prednisona 2,5 - 60,0 $\mathrm{mg} / \mathrm{kg} / \mathrm{dia})$ e $26(72,2 \%)$ pacientes de imunossupressores (azatioprina 2 - $3 \mathrm{mg} / \mathrm{kg} / \mathrm{dia}$, metotrexato 7,5 - $20 \mathrm{mg} / \mathrm{semana}$, talidomida $100 \mathrm{mg} / \mathrm{dia}$, micofenolato de mofetila 2 - 3 g/dia ou, ainda, pulsoterapia mensal com ciclofosfamida parenteral).

Todos usavam DFC, com o tempo de uso de 11,9 $\pm 5,1$ anos, dose cumulativa de 1.092,3 $\pm 476,6 \mathrm{~g}(456$ a $2.099 \mathrm{~g}$, mediana: $912 \mathrm{~g}$ ) e a dose diária ingerida, de acordo com o peso ideal, de 4,4 $\pm 0,5 \mathrm{mg} / \mathrm{kg}$ (Tabela 2). No entanto, quando

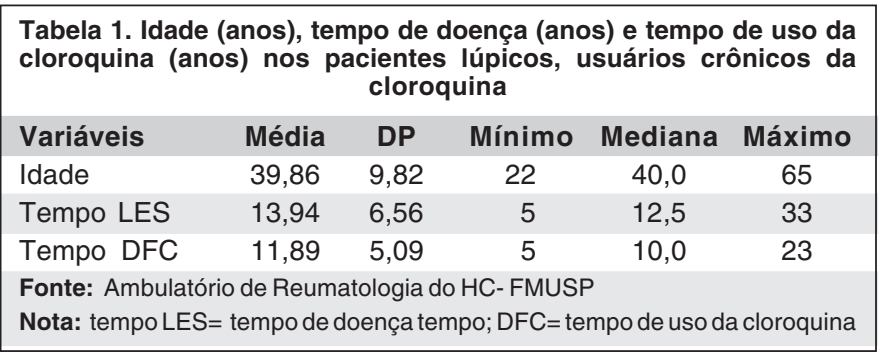

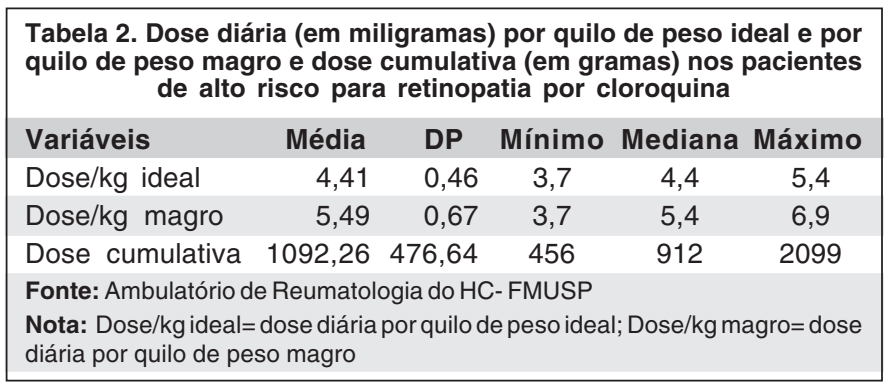

corrigido por peso magro, a dose diária ingerida foi de 5,5 \pm $0,7 \mathrm{mg} / \mathrm{kg}$ (Tabela 2 ).

A acuidade visual variou de $-0,2$ a 0,4 no olho direito (mediana: -0,04) e -0,2 a 0,5 no olho esquerdo (mediana: $-0,08)$, conforme mostra o quadro 1 .

A biomicroscopia da córnea foi considerada normal em apenas um paciente. O restante $(97,2 \%)$ apresentou algum grau de depósito corneal do DFC $(61,1 \%$ com ceratopatia ponteada e $36,1 \%$ com ceratopatia ponteada e infiltrados lineares). Todos os pacientes apresentaram pressão intraocular menor que $20 \mathrm{mmHg}$.

Os exames de biomicroscopia do fundo e retinografia mostraram alterações granulares na mácula em ambos os olhos de dois pacientes e rarefação do epitélio pigmentar da retina na mácula de ambos os olhos em outros dois pacientes (Figura 1). O restante dos pacientes $(88,9 \%)$ apresentou exame dentro da normalidade, sem alterações sugestivas de maculopatia tóxica.

O campo visual dos 10 graus centrais foi considerado normal em 61,1\% dos pacientes (Quadro 1). Entre os pacientes com alterações, houve $30,6 \%$ de escotomas absolutos paracentrais e/ou centrais, bilaterais, e 8,3\% de escotomas relativos bilaterais, centrais e/ou paracentrais (Quadros 1 e 2).

Alterações angiofluoresceinográficas foram observadas em três pacientes, que apresentaram defeitos em janela por rarefações na mácula em ambos os olhos (Figura 1). Não foram observadas alterações em $90,6 \%$ dos pacientes. O exame não foi realizado em quatro pacientes que referiram alergia a iodo.

O tempo de doença, a dose diária ingerida e o tempo de uso de DFC não se correlacionaram com o resultado dos exames realizados, exceto para angiofluoresceinografia (Gráfico 1). A dose cumulativa do DFC foi estatisticamente menor e significativa nos pacientes com alterações angiofluoresceinográficas em ambos os olhos (Gráfico 2).

A acuidade visual foi estatisticamente menor e significativa em ambos os olhos nos pacientes que apresentaram alterações detectadas na biomicroscopia do fundo e na angiofluoresceinografia (Gráfico 3).

Não houve correlação entre os achados do campo visual com os da acuidade visual, do mapeamento de retina, da angiofluoresceinografia ou da biomicroscopia.

\section{DISCUSSÃO}

Os vários efeitos benéficos atribuídos aos antimaláricos, em particular, nos pacientes com LES, contribuíram para a sua utilização de forma contínua e prolongada. Altas doses cumulativas da droga resultam em aumento de risco de maculopatia induzida por antimaláricos que, por sua vez, pode ser potencialmente irreversível. Até o presente momento, não existe um teste padrão para a detecção da maculopatia incipiente.

No presente estudo, foram avaliados os métodos diagnósticos mais comumente utilizados para avaliar a presença ou não de maculopatia induzida por DFC na população de pacientes lúpicos, usuários crônicos desta medicação. 


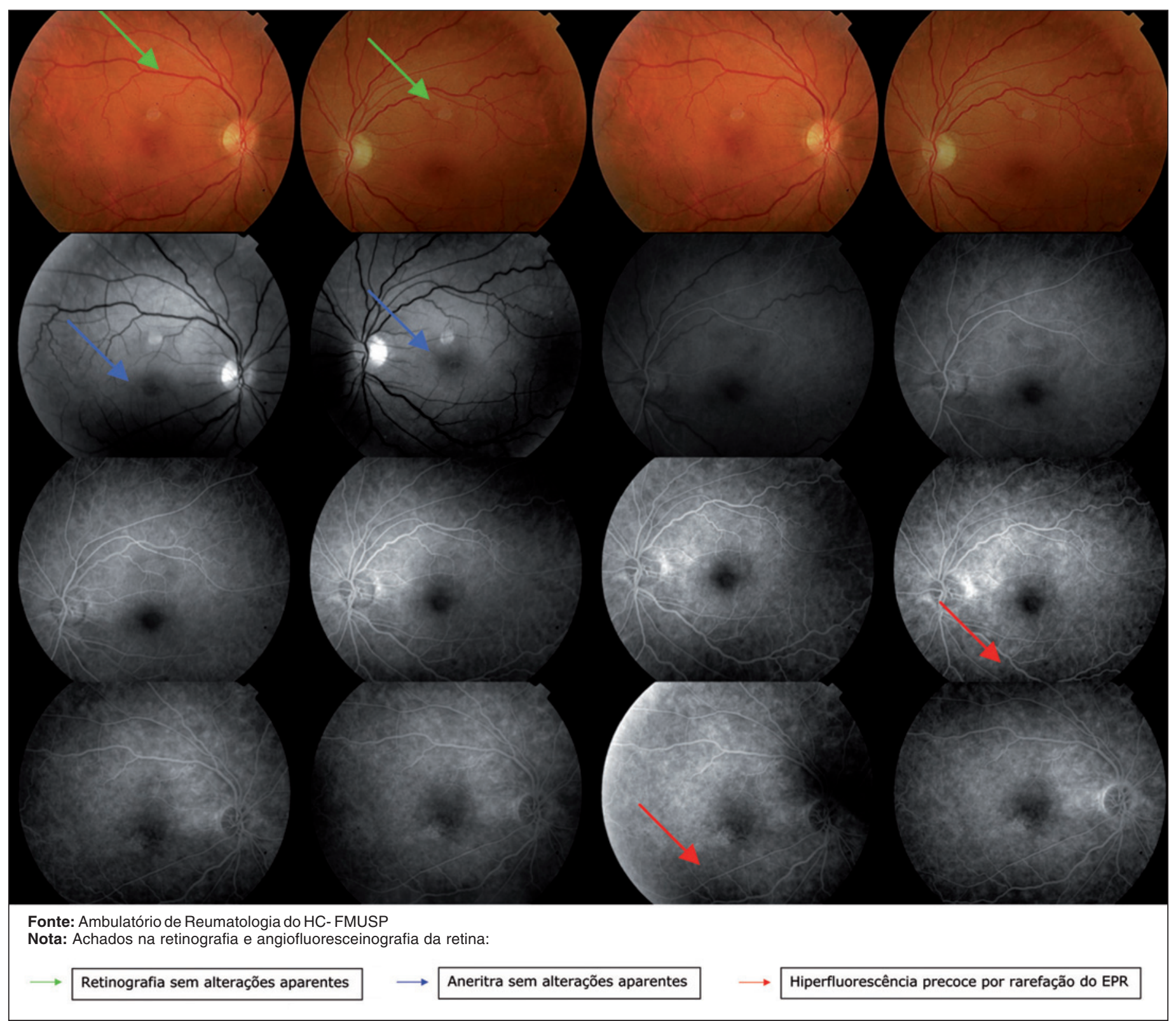

Figura 1 - Retinografia e angiofluoresceinografia da retina em usuário crônico da cloroquina (paciente 29) - abril de 2008

De modo geral, os pacientes apresentaram altas doses cumulativas do DFC. Embora tenha sido descrita maior prevalência de retinopatia nos usuários do DFC com dose cumulativa maior que $100 \mathrm{~g}^{(15)}$, isto não foi observado no presente estudo. Aliás, a dose cumulativa foi, inclusive, menor nos pacientes com alterações angiofluoresceinograficas e não mostrou relação com outros exames.

A literatura mostra que, inicialmente, a retinopatia foi associada ao uso crônico e altas doses cumulativas ${ }^{(16)}$ ou a altas doses diárias da droga ${ }^{(17)}$. Entretanto, observou-se a presença da alteração em pacientes com baixa exposição à $\operatorname{droga}{ }^{(18)} \mathrm{e}$, por outro lado, ausência de alterações em pacientes com uso prolongado e alta exposição ${ }^{(19)}$.
Também foi descrita maior incidência da retinopatia nos pacientes obesos, que são geralmente superdosados, uma vez que a cloroquina se deposita no espaço intracelular e não é absorvida pela gordura ${ }^{(20)}$. Por esta razão, o peso ideal passou a ser considerado no cálculo da dose diária segura, que foi definida como menor que $3,0 \mathrm{mg} / \mathrm{kg} / \mathrm{dia}^{(20)}$. Todos os pacientes apresentaram ingestão de dose diária maior que o recomendado, segundo o peso ideal e o peso magro. Entretanto, apesar da superdosagem, não houve maior prevalência da retinopatia nos pacientes com as doses diárias mais altas.

Com exceção dos pacientes com angiofluoresceinografia e biomicroscopia do fundo alterados, que apresentaram doses cumulativas e acuidade visual menores, deve-se observar que 


\begin{tabular}{|c|c|c|c|c|c|c|c|c|c|c|c|c|}
\hline \multirow[b]{2}{*}{$\mathbf{N}$} & \multirow[b]{2}{*}{ Idade } & \multirow[b]{2}{*}{ Sexo } & \multicolumn{2}{|c|}{ AV LogMAR } & \multicolumn{2}{|c|}{ Campo visual } & \multicolumn{2}{|c|}{ Bio do fundo } & \multicolumn{2}{|c|}{ Angio } & \multicolumn{2}{|c|}{ Bio da córnea } \\
\hline & & & OD & $\overline{O E}$ & OD & OE & OD & OE & OD & OE & OD & OE \\
\hline 1 & 35 & $\mathrm{~F}$ & 0,20 & 0,20 & $\mathrm{~nL}$ & $\mathrm{~nL}$ & $\mathrm{~nL}$ & $\mathrm{~nL}$ & $\mathrm{~nL}$ & $\mathrm{~nL}$ & $\mathrm{P}$ & $P$ \\
\hline 2 & 24 & $\mathrm{~F}$ & $-0,10$ & $-0,04$ & $\mathrm{~nL}$ & $n L$ & $\mathrm{~nL}$ & $\mathrm{~nL}$ & $\ldots$ & $\ldots$ & PL & PL \\
\hline 3 & 33 & $\mathrm{~F}$ & $\ldots$. & $\ldots$. & abs & abs & $\mathrm{nL}$ & $\mathrm{nL}$ & $\mathrm{nL}$ & $\mathrm{nL}$ & $P$ & $P$ \\
\hline 4 & 35 & $\mathrm{~F}$ & $-0,10$ & $-0,10$ & abs & abs & $\mathrm{nL}$ & $\mathrm{nL}$ & $\ldots$ & $\ldots$ & $P$ & $\mathrm{P}$ \\
\hline 5 & 37 & $\mathrm{~F}$ & $-0,10$ & $-0,10$ & $\mathrm{~nL}$ & $\mathrm{~nL}$ & $\mathrm{~nL}$ & $\mathrm{~nL}$ & $\mathrm{~nL}$ & $\mathrm{~nL}$ & $P$ & $\mathrm{P}$ \\
\hline 6 & 56 & $\mathrm{~F}$ & $-0,06$ & 0,04 & rel & abs & $\mathrm{nL}$ & $\mathrm{nL}$ & $\mathrm{nL}$ & $\mathrm{nL}$ & $P$ & $P$ \\
\hline 7 & 29 & $\mathrm{~F}$ & $-0,12$ & $-0,08$ & $\mathrm{~nL}$ & $\mathrm{~nL}$ & $\mathrm{~nL}$ & $\mathrm{~nL}$ & $\mathrm{~nL}$ & $\mathrm{~nL}$ & $P$ & $P$ \\
\hline 8 & 29 & $\mathrm{~F}$ & 0,00 & 0,00 & $\mathrm{~nL}$ & $\mathrm{~nL}$ & $\mathrm{~nL}$ & $\mathrm{~nL}$ & $\mathrm{~nL}$ & $\mathrm{~nL}$ & $P$ & $P$ \\
\hline 9 & 53 & $\mathrm{~F}$ & 0,14 & 0,22 & $\mathrm{~nL}$ & $\mathrm{~nL}$ & $\mathrm{~nL}$ & $\mathrm{~nL}$ & $\mathrm{~nL}$ & $\mathrm{~nL}$ & PL & PL \\
\hline 10 & 50 & $F$ & 0,06 & 0,06 & $\mathrm{~nL}$ & $\mathrm{~nL}$ & $\mathrm{~nL}$ & $\mathrm{~nL}$ & $\mathrm{~nL}$ & $\mathrm{~nL}$ & $\mathrm{~nL}$ & $\mathrm{~nL}$ \\
\hline 11 & 29 & $\mathrm{~F}$ & $-0,10$ & $-0,10$ & $\mathrm{~nL}$ & $\mathrm{~nL}$ & $\mathrm{~nL}$ & $\mathrm{~nL}$ & $\mathrm{~nL}$ & $\mathrm{~nL}$ & PL & PL \\
\hline 12 & 41 & $\mathrm{~F}$ & $-0,20$ & $-0,14$ & $\mathrm{~nL}$ & $\mathrm{~nL}$ & $\mathrm{~nL}$ & $\mathrm{~nL}$ & $\mathrm{~nL}$ & $\mathrm{~nL}$ & PL & PL \\
\hline 13 & 42 & $F$ & $-0,04$ & $-0,04$ & abs & abs & $\mathrm{nL}$ & $\mathrm{nL}$ & $\mathrm{nL}$ & $\mathrm{nL}$ & $P$ & $P$ \\
\hline 14 & 48 & $F$ & 0,20 & 0,12 & $\mathrm{~nL}$ & $\mathrm{~nL}$ & $\mathrm{~nL}$ & $\mathrm{~nL}$ & $\ldots$ & $\ldots$ & $P$ & $P$ \\
\hline 15 & 44 & F & 0,06 & $-0,04$ & abs & abs & $\mathrm{nL}$ & $\mathrm{nL}$ & $\mathrm{nL}$ & $\mathrm{nL}$ & $P$ & $P$ \\
\hline 16 & 43 & $\mathrm{~F}$ & $-0,02$ & $-0,12$ & abs & abs & $\mathrm{nL}$ & $\mathrm{nL}$ & $\mathrm{nL}$ & $\mathrm{nL}$ & PL & PL \\
\hline 17 & 37 & $\mathrm{~F}$ & $-0,06$ & 0,18 & $\mathrm{~nL}$ & $\mathrm{~nL}$ & $\mathrm{~nL}$ & $\mathrm{~nL}$ & $\mathrm{~nL}$ & $\mathrm{~nL}$ & $P$ & $P$ \\
\hline 18 & 39 & $\mathrm{~F}$ & $-0,16$ & $-0,04$ & $\mathrm{~nL}$ & $\mathrm{~nL}$ & $\mathrm{~nL}$ & $\mathrm{~nL}$ & $\mathrm{~nL}$ & $\mathrm{~nL}$ & PL & PL \\
\hline 19 & 46 & $\mathrm{~F}$ & $-0,14$ & $-0,18$ & abs & rel & $\mathrm{nL}$ & $\mathrm{nL}$ & $\ldots$ & $\ldots$ & $P$ & $P$ \\
\hline 20 & 24 & $F$ & 0,20 & 0,20 & abs & abs & $\mathrm{R}$ & $\mathrm{R}$ & $\mathrm{R}$ & $\mathrm{R}$ & $P$ & $P$ \\
\hline 21 & 52 & $\mathrm{~F}$ & 0,00 & $-0,20$ & abs & abs & $\mathrm{nL}$ & $\mathrm{nL}$ & $\mathrm{nL}$ & $\mathrm{nL}$ & PL & PL \\
\hline 22 & 31 & $F$ & $-0,20$ & $-0,20$ & abs & rel & $\mathrm{nL}$ & $\mathrm{nL}$ & $\mathrm{nL}$ & $\mathrm{nL}$ & $P$ & $P$ \\
\hline 23 & 53 & $\mathrm{~F}$ & 0,20 & 0,20 & $\mathrm{~nL}$ & $\mathrm{~nL}$ & $\mathrm{~nL}$ & $\mathrm{~nL}$ & $\mathrm{~nL}$ & $\mathrm{~nL}$ & PL & PL \\
\hline 24 & 41 & $\mathrm{~F}$ & 0,06 & $-0,08$ & $\mathrm{~nL}$ & $\mathrm{~nL}$ & $\mathrm{~nL}$ & $\mathrm{~nL}$ & $\mathrm{~nL}$ & $\mathrm{~nL}$ & $P$ & $P$ \\
\hline 25 & 42 & $\mathrm{~F}$ & 0,04 & 0,04 & $\mathrm{~nL}$ & $\mathrm{~nL}$ & $A G$ & $A G$ & $\mathrm{~nL}$ & $\mathrm{~nL}$ & $P$ & $P$ \\
\hline 26 & 37 & $\mathrm{~F}$ & 0,00 & $-0,10$ & rel & abs & $\mathrm{nL}$ & $\mathrm{nL}$ & $\mathrm{nL}$ & $\mathrm{nL}$ & $P$ & $P$ \\
\hline 27 & 46 & $\mathrm{~F}$ & 0,40 & 0,50 & abs & abs & $\mathrm{R}$ & $\mathrm{R}$ & $\mathrm{R}$ & $\mathrm{R}$ & PL & PL \\
\hline 28 & 42 & $\mathrm{~F}$ & $-0,04$ & $-0,08$ & rel & rel & $\mathrm{nL}$ & $\mathrm{nL}$ & $\mathrm{nL}$ & $\mathrm{nL}$ & PL & PL \\
\hline 29 & 50 & $\mathrm{~F}$ & 0,00 & 0,00 & abs & abs & $A G$ & $A G$ & $\mathrm{~nL}$ & $\mathrm{~nL}$ & $P$ & $P$ \\
\hline 30 & 43 & $F$ & $-0,08$ & $-0,08$ & $\mathrm{~nL}$ & $\mathrm{~nL}$ & $\mathrm{~nL}$ & $\mathrm{~nL}$ & $\mathrm{~nL}$ & $\mathrm{~nL}$ & PL & PL \\
\hline 31 & 38 & $\mathrm{~F}$ & $-0,16$ & $-0,16$ & $\mathrm{~nL}$ & $\mathrm{~nL}$ & $\mathrm{~nL}$ & $\mathrm{~nL}$ & $\mathrm{~nL}$ & $\mathrm{~nL}$ & $P$ & $P$ \\
\hline 32 & 65 & $\mathrm{~F}$ & $-0,06$ & $-0,04$ & $\mathrm{~nL}$ & $\mathrm{~nL}$ & $\mathrm{~nL}$ & $\mathrm{~nL}$ & $\mathrm{~nL}$ & $\mathrm{~nL}$ & $P$ & $P$ \\
\hline 33 & 26 & $M$ & $-0,10$ & $-0,10$ & $\mathrm{~nL}$ & $\mathrm{~nL}$ & $\mathrm{~nL}$ & $\mathrm{~nL}$ & $\mathrm{~nL}$ & $\mathrm{~nL}$ & PL & PL \\
\hline 34 & 22 & $\mathrm{~F}$ & $-0,20$ & $-0,20$ & $\mathrm{~nL}$ & $\mathrm{~nL}$ & $\mathrm{~nL}$ & $\mathrm{~nL}$ & $\mathrm{~nL}$ & $\mathrm{~nL}$ & $P$ & $P$ \\
\hline 35 & 36 & $M$ & 0,00 & $-0,10$ & $\mathrm{~nL}$ & $\mathrm{~nL}$ & $\mathrm{~nL}$ & $\mathrm{~nL}$ & $\mathrm{~nL}$ & $\mathrm{~nL}$ & PL & PL \\
\hline 36 & 37 & $\mathrm{~F}$ & 0,00 & 0,00 & $\mathrm{~nL}$ & $\mathrm{~nL}$ & $\mathrm{~nL}$ & $\mathrm{~nL}$ & $R$ & $\mathrm{R}$ & $P$ & $\mathrm{P}$ \\
\hline \multicolumn{13}{|c|}{ Fonte: Ambulatório de Reumatologia do HC- FMUSP } \\
\hline $\begin{array}{l}\mathrm{AV}= \\
\mathrm{abs} \\
\mathrm{PL}=\end{array}$ & $\begin{array}{l}\text { uidade vis } \\
\text { scotomas } \\
\text { iltrados }\end{array}$ & $\begin{array}{l}\text { Bio= bior } \\
\text { olutos; re } \\
\text { ormes e I }\end{array}$ & $\begin{array}{l}\text { copia; A } \\
\text { otomas } \\
\text {; }\end{array}$ & $\begin{array}{l}\mathrm{D}=\text { angio } \\
\text { tivos; } \mathrm{R}=\end{array}$ & $\begin{array}{l}\text { grafia } \\
\text { arefa }\end{array}$ & $\begin{array}{l}=\text { olhc } \\
\text { do epi }\end{array}$ & $\begin{array}{l}=\text { olh } \\
\operatorname{tar} \mathrm{d}\end{array}$ & $\begin{array}{l}\text { uerdo; } \\
\text { la; AG }\end{array}$ & $\begin{array}{l}\text { emini } \\
\text { es gra }\end{array}$ & $\begin{array}{l}\mathrm{M}=\mathrm{se} \\
\text { lares; }\end{array}$ & $\begin{array}{l}\text { ulino; nL } \\
\text { ados pu }\end{array}$ & $\begin{array}{l}\text { rmal; } \\
\text { rmes; }\end{array}$ \\
\hline
\end{tabular}

o grupo apresentou valores semelhantes de doses diárias e cumulativas nos pacientes com e sem a maculopatia (medianas muito próximas, Tabela 2).

Assim, o desenvolvimento ou não da retinopatia, na presença de altas doses diárias e cumulativas, pode estar relacionado a fatores como a suscetibilidade individual e a própria doença, cuja interferência no metabolismo retiniano facilita o aparecimento da retinopatia ${ }^{(21)}$.

A biomicroscopia da córnea mostrou alterações em quase todo o grupo, o que também foi observado por Easterbrooke em cerca de $90 \%$ dos seus pacientes ${ }^{(22)}$. Os depósitos corneanos localizados no epitélio e no estroma subepitelial são comuns, geralmente assintomáticos, e desaparecem progressivamente em seis a oito semanas ${ }^{(23)}$. As alterações observadas pela biomicroscopia com retroiluminação e sob midríase são os depósitos ponteados difusos ou as linhas pigmentadas (córnea Verticilatta $)^{(24-25)}$. Raramente ocorre comprometimento visual ${ }^{(26)}$.

Não houve relação entre a presença de ceratopatia e o desenvolvimento de maculopatia, como já observado por outros autores ${ }^{(27)}$. A presença de deposição corneana não significa existência da retinopatia ${ }^{(28)}$, mas está relacionada ao acúmulo do DFC nos tecidos e à superdosagem ${ }^{(29)}$.

A incidência da retinopatia varia de acordo com a definição e os exames usados para o seu diagnóstico. Considerando que nos 


\begin{tabular}{|c|c|c|c|c|c|c|}
\hline \multirow[t]{3}{*}{$\mathbf{N}$} & \multicolumn{2}{|c|}{ AV IogMAR } & \multicolumn{4}{|c|}{ Campo visual } \\
\hline & \multirow[t]{2}{*}{ OD } & \multirow[t]{2}{*}{$\overline{O E}$} & \multicolumn{2}{|c|}{ OD } & \multicolumn{2}{|c|}{ OE } \\
\hline & & & Tipo & Local & Tipo & Local \\
\hline 3 & $\ldots$ & $\ldots$ & Absolutos & Sup 6 & Absolutos & Sup $4 / 6$ \\
\hline 4 & $-0,10$ & $-0,10$ & Absolutos & NS $4 / 6$ & Absolutos & Sup 8 \\
\hline 6 & $-0,06$ & 0,04 & Relativos & Sup 6 & Absolutos & NS $4 / 6$ \\
\hline 13 & $-0,04$ & $-0,04$ & Absolutos & Sup $2 / 4$ & Relativos & Sup 2/4 \\
\hline 15 & 0,06 & $-0,04$ & Absolutos & Sup e NS 4/6/8 & Absolutos & Sup e TS 6/8 \\
\hline 16 & $-0,02$ & $-0,12$ & Absolutos & Sup 2/4/6/8; Inf 2 & Absolutos & Sup 2/4/8/10; Inf 2 \\
\hline 19 & $-0,14$ & $-0,18$ & Absolutos & Sup 2 & Relativos & Sup $4 / 6$ \\
\hline 20 & 0,20 & 0,20 & Absolutos & Sup 2/4/8 & Absolutos & Sup 2; NS 8 \\
\hline 21 & 0,00 & $-0,20$ & Absolutos & Sup 4/6; NS 2/4 & Absolutos & NS 4 \\
\hline 22 & $-0,20$ & $-0,20$ & Absolutos & TS $2 / 4$ & Relativos & NS 4 \\
\hline 26 & 0,00 & $-0,10$ & Relativos & Sup 4 & Absolutos & Sup 2; Inf 2 \\
\hline 27 & 0,40 & 0,50 & Absolutos & Sup 2/4/6; Inf 2 & Absolutos & Sup $2 / 4 ;$ Inf 2/4 \\
\hline 28 & $-0,04$ & $-0,08$ & Relativos & Sup $4 / 6$ & Relativos & Sup $4 / 6$ \\
\hline 29 & 0,00 & 0,00 & Absolutos & Sup $2 / 4$ & Absolutos & Sup $2 / 4 / 6$ \\
\hline \multicolumn{7}{|c|}{ Fonte: Ambulatório de Reumatologia do HC- FMUSP } \\
\hline \multicolumn{7}{|c|}{$\begin{array}{l}\text { AV } \log M A R=\text { acuidade visual em logMAR; } O D=\text { olho direito; } O E=\text { olho esquerdo; Tipo= profundidade do defeito no campo visual; Local= localização do defeito no campo } \\
\text { visual (2,4,6,8 ou } 10 \text { graus); Absolutos= escotomas absolutos; Relativos= escotomas relativos; Sup= campo superior; Inf= campo inferior; NS= campo nasal superior; } \\
\text { TS= campo temporal superior }\end{array}$} \\
\hline
\end{tabular}

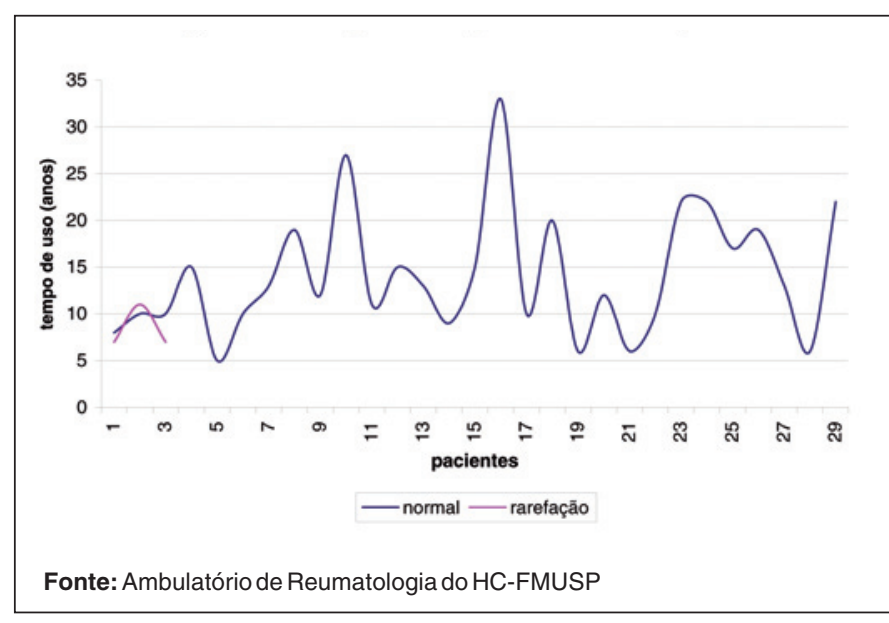

Gráfico 1 - Distribuição dos achados na angiofluoresceinografia da retina segundo tempo de uso, nos usuários crônicos da cloroquina - 2008

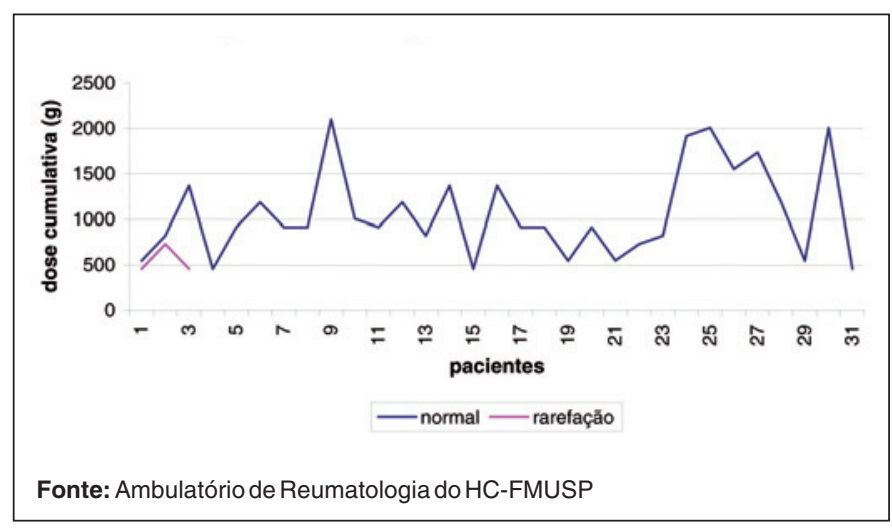

Gráfico 2 - Distribuição dos achados na angiofluoresceinografia segundo dose cumulativa, nos usuários crônicos da cloroquina - 2008

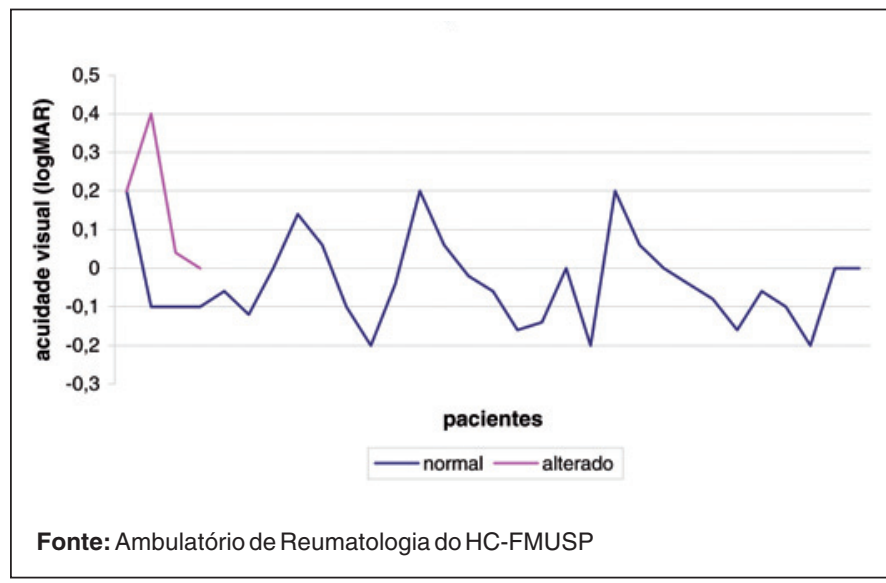

Gráfico 3 - Distribuição da acuidade visual segundo os achados na biomicroscopia indireta para o olho direito, nos usuários crônicos da cloroquina - 2008

pacientes usuários de cloroquina em geral (com baixo e alto risco), a retinopatia precoce pode chegar a $16 \%^{(7)}$, é possível deduzir que a prevalência da mesma nos pacientes usuários crônicos e, portanto, com alto risco para a retinopatia, seja maior.

Utilizou-se como critério diagnóstico para a presença da retinopatia a presença de defeito adquirido, central ou paracentral, bilateral e reprodutível no campo visual ${ }^{(7)}$. A maioria dos exames de biomicroscopia do fundo e angiofluoresceinografia foi considerada normal, enquanto o campo visual mostrou alterações em aproximadamente $40 \%$. Isto sugere, segundo a definição de Easterbrooke ${ }^{(7)}$, que estes pacientes apresentam retinopatia precoce. Do mesmo modo, os pacientes com angiofluoresceinografia e biomicroscopia do fundo alterados, que apresentaram acuidades visuais menores e de- 


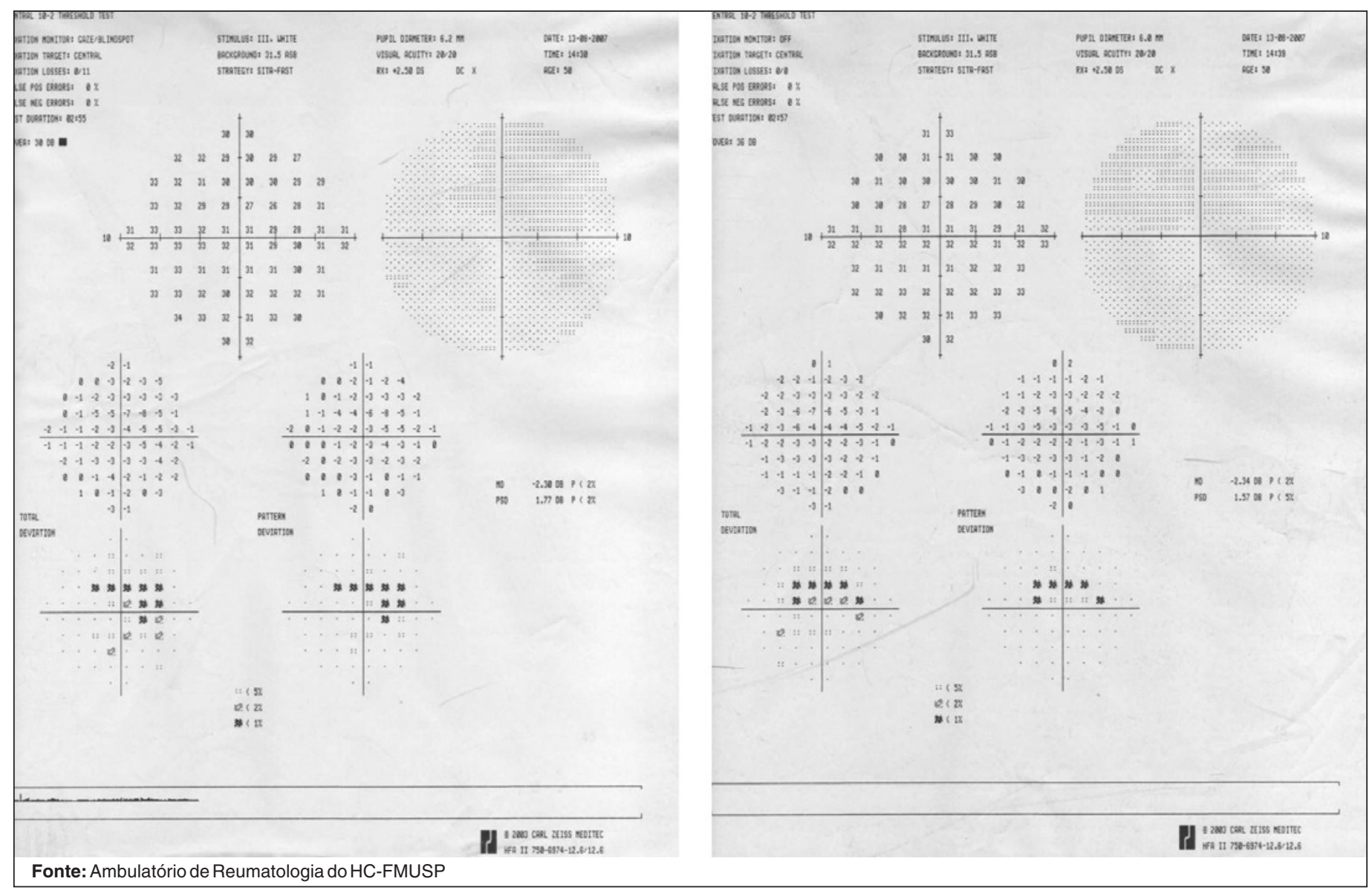

Figura 2 - Campimetria visual (Humphrey, Macula 10.2, mira branca, SITAFAST) em usuário crônico da cloroquina (paciente 29) - abril de 2008

feitos típicos no campo visual (pacientes 20 e 27) podem ser considerados portadores da retinopatia estabelecida (Quadro 1 , Figuras 1 e 2). Outros pacientes apresentaram escotomas centrais sem alteração importante da acuidade visual (pacientes 13, 16, 19, 21, 22, 26, 29. Quadros 1 e 2), o que mostra que o acometimento da acuidade visual pode ocorrer apenas nas fases mais avançadas.

É possível, entretanto, que alterações funcionais próprias do LES tenham interferência nos achados dos exames. A presença de retinopatia secundária ao próprio LES, que poderia ser responsável pelos diversos achados nos exames realizados, foi excluída pelos exames de biomicroscopia binocular indireta e de angiofluoresceinografia da retina. Sabe-se que alterações funcionais podem estar presentes nos pacientes com artrite reumatóide mesmo antes do uso dos antimaláricos $^{(30)}$, o que também é questionado nos pacientes lúpicos. Deve-se considerar ainda que outras drogas utilizadas no tratamento destes pacientes também podem causar toxicidade retiniana e neuropatia óptica, como é o caso dos imunossupressores ${ }^{(31-32)}$, o que pode ter contribuído para a alta prevalência da retinopatia neste grupo.

Apesar de apresentar maior toxicidade que a hidroxicloroquina ${ }^{(33)}$, o DFC é altamente benéfico aos pacientes lú- $\operatorname{picos}^{(1-6)}$ e apresenta baixo custo. Por seus excelentes resultados terapêuticos, o uso do DFC tende a ser cada vez mais generalizado e prolongado nestes pacientes.

A classificação dos pacientes segundo o risco de desenvolverem a retinopatia seleciona os pacientes que devem ser avaliados em intervalos menores ${ }^{(9)}$. Entretanto, o presente estudo mostrou que os fatores de risco não puderam selecionar os pacientes suscetíveis. A alta prevalência da retinopatia encontrada nos usuários crônicos do DFC justifica a avaliação em intervalos ainda menores que os propostos atualmente.

A maioria dos exames indicados para rastreamento e seguimento da retinopatia por antimaláricos também não foram capazes de diagnosticar a maioria das alterações detectadas pelos campos visuais. A inexistência de um teste padrão para o rastreamento da retinopatia precoce e a observação da progressão da maculopatia tardia mesmo após a interrupção da droga $^{(34-36)}$ tornam necessário o estudo de novos exames para o rastreamento.

Exames possivelmente mais sensíveis, como o ERG multifocal, OCT e GDx ainda requerem estudos sobre a sua sensibilidade. Até o presente momento não há indícios de que estes exames sejam mais sensíveis que a campimetria visual ${ }^{(37)}$. A realização de exames em intervalos menores e a realização do 
exame de campo visual devem ser considerados nestes pacientes, mesmo quando não há suspeita clínica.

\section{ABSTRACT}

Purpose: To evaluate different diagnostic methods for high risk chloroquine retinopathy due to prolonged use of chloroquine (more than 5 years) by systemic lupus erythematosus patients. Methods: Seventy-two eyes of 36 consecutive patients, followed in the Division of Rheumatology, School of Medicine, University of São Paulo, were analyzed from July 2007 to April 2008. Demographic and clinical data were evaluated in order to study risk factors and to compare the following different ophthalmological methods: visual acuity, biomicroscopy, fundus examination, retinography, fluorescein angiogram, visual field test and, color vision tests. Results: From 36 patients, 34 (94.4\%) were female. The mean age was $39.9 \pm 9.8$ years and the disease duration was $13.9 \pm 6.6$ years. Besides chronic use of chloroquine, patients also showed high daily and cumulative doses. These high risk factors were not related to a higher retinopathy prevalence. Visual field showed $38.9 \%$ of retinopathy prevalence. Other ophthalmological methods failed in detecting most cases. Conclusion: High prevalence of retinopathy in high risk patients was observed by visual field test, but other ophthalmological methods failed in detecting alterations. Ophthalmological assessment of these patients should include visual field, even in the absence of clinical alterations.

Keywords: Retina/pathology; Retina/drug effects; Chloroquine/ therapeutic use; Lupus erythematosus, systemic; Risk factors

\section{REFERÊNCIAS}

1. Costedoat-Chalumeau N, Amoura Z, Hulot JS, Hammoud HA, Aymard G, Cacoub $\mathrm{P}$, et al. Low blood concentration of hydroxychloroquine is a marker for and predictor of disease exacerbations in patients with systemic lupus erythematosus. Arthritis Rheum. 2006;54(10):3284-90. Comment in: Arthritis Rheum. 2006;54(10):3068-70.

2. de Carvalho JF, Borba EF, Viana VS, Bueno C, Leon EP, Bonfá E. Antilipoprotein lipase antibodies: a new player in the complex atherosclerotic process in systemic lupus erythematosus? Arthritis Rheum. 2004;50(11):3610-5.

3. Edwards MH, Pierangeli S, Liu X, Barker JH, Anderson G, Harris EN. Hydroxychloroquine reverses thrombogenic properties of antiphospholipid antibodies in mice. Circulation. 1997;96(12):4380-4

4. Hansen EH, Jessing P, Lindewald H, Ostergaard P, Olesen T, Malver EI. Hydroxychloroquine sulphate in prevention of deep venous thrombosis following fracture of the hip, pelvis, or thoracolumbar spine. J Bone Joint Surg Am. 1976; 58(8):1089-93.

5. Frostegard J. SLE, atherosclerosis and cardiovascular disease. J Intern Med. 2005; 257(6):485-95. Comment in: J Intern Med. 2005;258(6):584; author reply 585-6.

6. Chogle AR, Chakravarty A. Cardiovascular events in systemic lupus erythematosus and rheumatoid arthritis: emerging concepts, early diagnosis and management. J Assoc Physicians India. 2007;55:32-40.

7. Easterbrook M. Ocular effects and safety of antimalarial agents. Am J Med. 1988; 85(4A):23-9.
8. Easterbrook M. Current concepts in monitoring patients on antimalarials. Aust N Z J Ophthalmol. 1998;26(2):101-3.

9. Marmor MF, Carr RE, Easterbrook M, Farjo AA, Mieler WF; American Academy of Ophthalmology. Recommendations on screening for chloroquine and hydroxychloroquine retinopathy: a report by the American Academy of Ophthalmology. Ophthalmology. 2002;109(7):1377-82.

10. Hobbs HE, Calnan CD. The ocular complications of chloroquine therapy. Lancet. 1958;1(7032):1207-9.

11. Amsler M. L'examen qualitif de la fonction maculaire. Ophthalmologica. 1947; 114:248-61.

12. Hochberg MC. Updating the American College of Rheumatology revised criteria for the classification of systemic lupus erythematosus. Arthritis Rheum. 1997; 40(9):1725. Comment in: Arthritis Rheum. 1998;41(4):751. Arthritis Rheum. 1998;41(7):1326-7. Arthritis Rheum. 1999;42(3):585.

13. Shah B, Sucher K, Hollenbeck CB. Comparison of ideal body weight equations and published heigth-weigth tables with body mass index tables for healthy adults in the United States. Nutr Clin Pract. 2006;21(3):312-9.

14. James WPT. Research on obesity. A report of the DHSS/MRC Group. London: HMSO; 1976

15. Nylander U. Ocular damage in chloroquine therapy. Acta Ophthalmol (Copenh). 1967;(Suppl)92:1-71.

16. Bernstein HN, Ginsberg J. The pathology of chloroquine retinopathy. Arch Ophthalmol. 1964;71:238-45.

17. Carr RE, Gouras P, Gunkell RD. Chloroquine retinopathy. Early detection by retinal threshold test. Arch Ophthalmol. 1966;75(2):171-8.

18. Nylander U. Ocular damage in chloroquine therapy. Acta Ophthalmol (Copenh). 1966;44(3):335-48.

19. Johnson MW, Vine AK. Hydroxychloroquine therapy in massive total doses without retinal toxicity. Am J Ophthalmol. 1987;104(2):139-44.

20. Mackenzie AH. Dose refinements in long-term therapy of rheumatoid arthritis with antimalarials. Am J Med. 1983;75(1A):40-5.

21. Shroyer NF, Lewis RA, Lupski JR. Analysis of the ABCR (ABCA4) gene in 4-aminoquinoline retinopathy: is retinal toxicity by chloroquine and hydroxychloroquine related to Stargardt disease? Am J Ophthalmol. 2001;131(6):761-6.

22. Arden GB, Kolb H. Electrophysiological investigations in retinal metabolic disease: their range and application. Exp Eye Res. 1964;3:334-47.

23. Easterbrook M. Is corneal deposition of antimalarial any indication of retinal toxicity? Can J Ophthalmol. 1990;25(5):249-51.

24. Hobbs HE, Eadie SP, Somerville F. Ocular lesions after treatment with chloroquine. Br J Ophthalmol. 1961;45(4):284-97.

25. Calkins LL. Corneal epithelial changes occurring during chloroquine (aralen) therapy. AMA Arch Ophhthalmol. 1958;60(6):981-8.

26. Seiler KU, Thiel HJ, Wassermann O. [Chloroquine keratopathy as an example of drug-induced phospholipidosis (contribution to the pathogenesis of cornea verticillata). (author's transl)]. Klin Monastbl Augenheilkd. 1997;170(1):64-73. German.

27. Easterbrook M. Ocular effects and safety of antimalarial agents. Am J Med. 1988;85(4A):23-9.

28. Bernstein H, Zvaifler N, Rubin M, Mansour AM. The ocular deposition of chloroquine. Invest ophlthmol Vis Sci. 1963;2:384-92.

29. Easterbrook M. Is corneal deposition of antimalarial any indication of retinal toxicity? Can J Ophthalmol. 1990;25(5):249-51.

30. Gouras P, Gunkel RD. The EOG in chloroquine and other retinopathies. Arch Ophthalmol. 1963;70:629-39.

31. Burns CA. Indomethacin, reduced retinal sensitivity, and corneal deposits. Am J Ophthalmol. 1968;66(5):825-35.

32. Ponjavic V, Gränse L, Stigmar EB, Andréasson S. Reduced full-field electroretinogram (ERG) in a patient treated with methotrexate. Acta Ophthalmol Scand. 2004;82(1):96-9. Comment in: Acta Ophthalmol Scand. 2004;82(5): 625.

33. Aviña-Zubieta JA, Galindo-Rodriguez G, Newman S, Suarez-Almazor ME, Russell AS. Long-term effectiveness of antimalarial drugs in rheumatic diseases. Ann Rheum Dis. 1998;57(10):582-7.

34. Easterbrooke M. Long-term course of antimalarial maculopathy after cessation of treatment. Can J Ophthalmol. 1992;27(5):237-9.

35. Brinkley JR Jr, Dubois EL, Ryan SJ. Long-term course of chloroquine retinopathy after cessation of medication. Am J Ophthalmol. 1979;88(1):1-11.

36. Ehrenfeld M, Nesher R, Merin S. Delayed-onset chloroquine retinopathy. Br J Ophthalmol. 1986;70(4):281-3

37. Marmor MF. The dilemma of hydroxychloroquine screening: new information from the multifocal ERG. Am J Ophthalmol. 2005;140(5):894-5. Comment on: Am J Ophthalmol. 2005;140(5):794-807. 